\title{
Quantum dot formation in InGaN/GaN quantum well structures with silicon doping and the mechanisms for radiative efficiency improvement
}

\author{
Yung-Chen Cheng ${ }^{1}$, Shih-Wei Feng ${ }^{1}$, En-Chiang Lin $^{1}$, Chih-Chung Yang ${ }^{*}$, , \\ Cheng-Hua Tseng ${ }^{2}$, Cheng $\mathrm{Hsu}^{2}$, and Kung-Jen $\mathrm{Ma}^{3}$ \\ ${ }^{1}$ Department of Electrical Engineering and Graduate Institute of Electro-Optical Engineering, National \\ Taiwan University, 1, Roosevelt Road, Sec. 4, Taipei, Taiwan \\ 2 Department of Mechanical Engineering, Chung Cheng Institute of Technology, Tahsi, Taoyuan, Taiwan \\ ${ }^{3}$ Department of Mechanical Engineering, Chung Hua University, Hsinchu, Taiwan
}

Received 30 September 2002, accepted 2 December 2002

Published online 23 June 2003

PACS 68.65.Hb, 78.67.De, 78.67.Hc

Material and optical analyses of three InGaN/GaN quantum well (QW) samples with different silicon doping conditions were conducted. Quantum dot (QD) structures were observed in samples of silicon doping either in barriers or wells. Optical characterization indicated that quantum-confined Stark effect (QCSE) has been tremendously reduced, particularly in the barrier-doped sample. However, contrary to the interpretation in the past, the major mechanism for QCSE reduction is due to strain relaxation, instead of carrier screening, in conjunction with the formation of QD structures. Such a conclusion is supported by the smaller change of Stokes shift upon silicon doping in wells. In the well-doped sample, besides strain relaxation, enhanced carrier localization might represent another important mechanism for photon emission improvement.

1 Introduction Silicon doping in InGaN/GaN quantum well $(\mathrm{QW})$ structures for improving photon emission efficiency were reported [1-3]. However, the real material microstructures and their connections with optical properties in silicon-doped InGaN/GaN QW structures have never been well discussed. Information of material microstructures can provide direct evidence for interpreting the results of optical characterization.

Our research emphasized the microstructure alternations with silicon doping and their connections with optical properties. In particular, the influences of induced strain relaxation and carrier confinement on optical characteristics were studied. The results show that samples with silicon doping tend to form 0-D clusters, i.e., quantum dots (QDs). Consequently, strain relaxation due to microstructure alteration upon silicon doping may play a key role in improving photon emission efficiency, particularly in a barrier-doped sample. On the other hand, the enhanced carrier confinement may be the major cause for increasing photon emission intensity in a well-doped sample.

2 Sample preparation Three InGaN/GaN QW samples of the same geometry and nominal indium content were prepared with MOCVD growth on sapphire substrate. They all consisted of five QW periods with $3 \mathrm{~nm}$ in well width and $7 \mathrm{~nm}$ in barrier width. A $20 \mathrm{~nm} \mathrm{GaN}$ cap layer was grown on top of the five QW layers. The nominal indium content was about $15 \%$. The growth temperatures were 1050 and

* Corresponding author: e-mail: ccy@cc.ee.ntu.edu.tw, Phone: 886-23657624, Fax: 886-23652637 


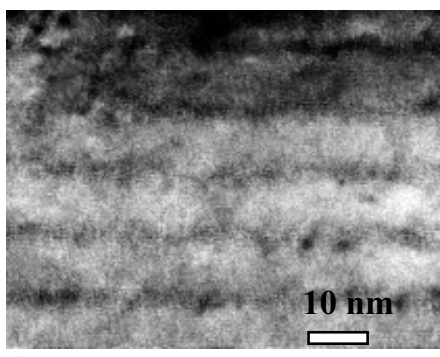

a)

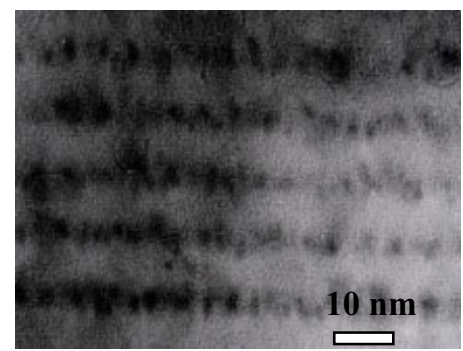

b)

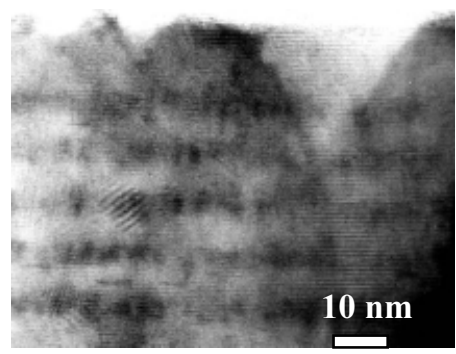

c)

Fig. 1 HRTEM images of the sample: a) undoped, b) barrier-doped, and c) well-doped.

$740{ }^{\circ} \mathrm{C}$ for $\mathrm{GaN}$ and InGaN, respectively. The doping concentrations are the same $\left(5 \times 10^{18} \mathrm{~cm}^{-3}\right)$ either in barriers or in wells. The un-doped, barrier-doped and well-doped samples are referred to as samples A, $\mathrm{B}$ and $\mathrm{C}$, respectively.

3 Material analysis results Figures $1 \mathrm{a}, \mathrm{b}$, and c show the HRTEM images of samples A, B and C, respectively. Although the five QWs can be clearly identified in Fig. 1a (sample A, undoped), the image here indicates that indium composition extensively spreads from wells into barriers to form clusters of quite large and irregular sizes. On the contrary, as shown in Fig. 1b, in the barrier-doped sample (B) nano-scale clusters (QDs) with more regular sizes and shapes are arranged along the designated QW layers. The sizes of the dots range from 3 to $5 \mathrm{~nm}$. Such structures are expected to strongly localize excitons, preventing from non-radiative recombination outside clusters. Although the quasi-regular arrays of QD in the well-doped sample (C) is not as clear as the barrier-doped sample, sharp clusters can be clearly seen in sample C (see Fig. 1c). From the optical and material analyses of similar samples in other research [4], we learned that the QD structures in samples B and C typically result in higher photon emission efficiencies, which are to be confirmed in the following discussions of optical characterization.

4 Optical characterization results Figure 2 a shows the PL spectral peak positions as functions of temperature of the three samples. The blue shift $(73 \mathrm{meV}$ at $10 \mathrm{~K})$ of PL peak in sample B compared with the undoped sample (A) has been widely observed. The PL peaks of the well-doped sample (C) approximately coincide with those of the undoped sample (A), except the small red shifts below $80 \mathrm{~K}$ and above $200 \mathrm{~K}$. All the curves in Fig. 2 a show S-shape variations of PL peak, which are typical behaviors of such clustered and strained structures. Figure $2 \mathrm{~b}$ shows the PL spectra of the three samples at $10 \mathrm{~K}$. Figure 3 shows temperature-dependent integrated PL intensities of the three samples. One can see that silicon doping results in relatively higher photon emission intensity (based on temperature variation), particularly in the temperature range above $200 \mathrm{~K}$. With silicon doping, the normalized luminescence intensity is about two orders of magnitude higher than the undoped sample at the room temperature. It is
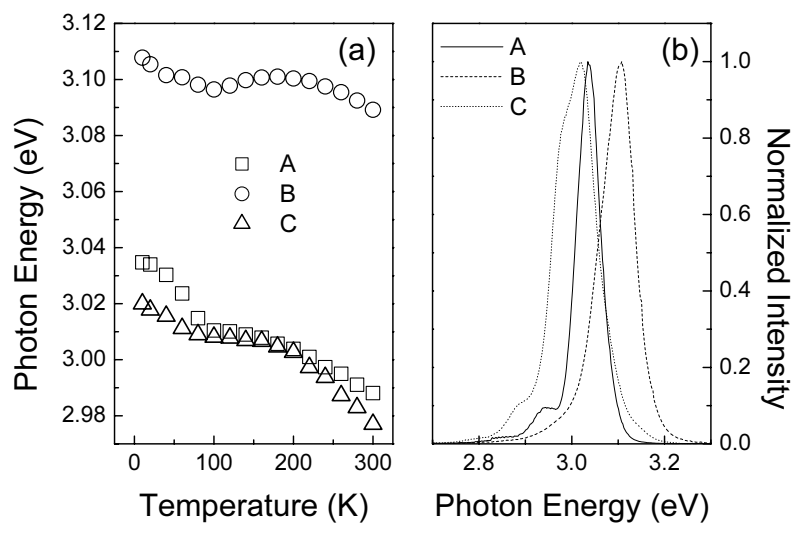

Fig. 2. (a) PL peak energies as functions of temperature for the three samples; (b) PL spectra of the three samples at $10 \mathrm{~K}$. 


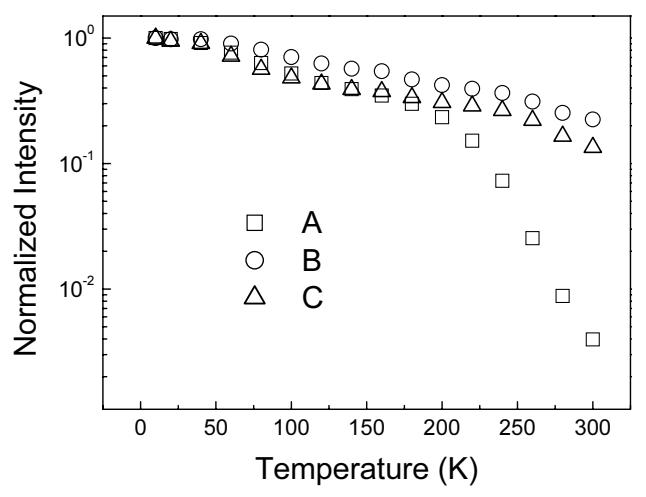

Fig. 3 Normalized PL intensity as functions of temperature for the three samples.

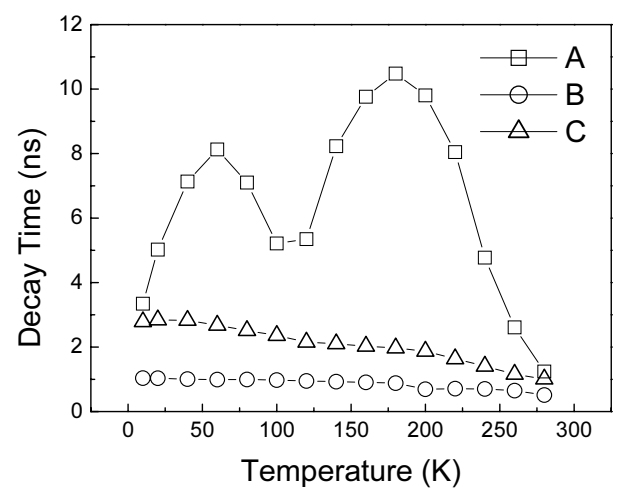

Fig. 4 PL decay times as functions of temperature for the three samples.

interesting to note that the normalized integrated PL intensity variations with temperature of all the samples can generally be divided into three stages with the boundaries around 80 and $200 \mathrm{~K}$.

Figure 4 shows the temperature-dependent decay time variations at individual PL peaks of the three samples. The reduction of PL decay time upon silicon doping is more significant in the barrier-doped sample than the well-doped one. Two peaks with temperature variation can be clearly seen in sample A. Such peak features become less prominent in samples B and C. The two peaks of PL decay time appear at 60 and $180 \mathrm{~K}$ (denoted by $T_{1}$ and $T_{2}$, respectively), which are roughly consistent with the counterparts in Fig. 3, implying that they have certain physical meanings.

5 Discussion With the strain relaxed and the quasi-regularly arranged QDs formed, QCSE within dotlike clusters is reduced, leading to PL blue shift and enhanced radiative transition rate. These predictions have been confirmed with the results shown in Figs. 2-4. In the past, the above-mentioned optical phenomena observed in barrier silicon-doped samples were usually attributed to carrier screening of the piezoelectric fields upon silicon doping. However, the comparison between the results of samples B and $\mathrm{C}$ indicates that the carrier screening effect may play a minor role in reducing QCSE in our samples. Because the large $(\sim 114 \mathrm{meV})$ and small $(\sim 19 \mathrm{meV})$ decreases of Stokes shift in barrier-doped and welldoped samples, respectively, compared to the un-doped one, were observed at $10 \mathrm{~K}$ (figures not shown in this paper), the reduction of piezoelectric fields may not be the main cause leading to the increase of emission intensity in the well-doped sample. The significant blue shift of PL peak in sample B (see Fig. 2a) can be attributed to the effect of quantum confinement besides the reduction of QCSE. The insignificant shift of PL peak in sample C may originate from the interplay of several different mechanisms, including the reduction of QCSE (blue shift trend), impurity level recombination (red shift), and the indium clustering effect (red shift trend). In the process of further indium clustering upon silicon doping, carrier potential is reduced and carriers are more strongly localized. Also, the contribution of impurity level recombination may play an important role in sample $\mathrm{C}$. The characteristic temperatures of $T_{1}$ and $T_{2}$ were often observed in similar QW structures in our research. Below $T_{1}$, carriers can move around near the center of a cluster and relax into the potential minimum such that the PL decay time increases and PL intensity almost keeps constant with increasing temperature. Between $T_{1}$ and $T_{2}$, carriers receive certain thermal energy and they can move essentially freely within a cluster or transport between coupled clusters. In this situation, carriers may be consumed through defects around clusters. Therefore, PL intensity decreases. Also, PL decay time may first decrease due to the aforementioned defects. However, with slightly more thermal energy, carriers can overcome certain barriers and be trapped by more strongly localized states. Hence the PL decay time is increased again. Beyond $T_{2}$, carriers can escape from localized states and PL intensity decreases more significantly with temperature. Also, PL decay time decreases monotonically with temperature. These processes of carrier dynamics may dominate in different temperature ranges such that the results in Figs. 2-4 can be observed. 
6 Conclusions In summary, from HRTEM images, QDs were observed in samples of silicon doping either in barriers or wells. Although carrier screening has been used for explaining the reduction of QCSE, it was believed that the major mechanism for QCSE reduction was due to strain relaxation in conjunction with the formation of QD structures. Such a conclusion is supported by the smaller change of Stokes shift upon silicon doping in wells. In this sample, the major mechanism for photon emission improvement might be the enhanced carrier localization effect.

Acknowledgements This research was supported by National Science Council, The Republic of China, under the grants of NSC 90-2112-M-002-052, NSC 90-2215-E-002-027, and NSC 90-2215-E-002-041.

\section{References}

[1] M. S. Minsky, S. Chichibu, S. B. Fleischer, A. C. Abare, J. E. Bowers, E. L. Hu, S. Keller, U. K. Mishra, and S. P. DenBaar, Jpn. J. Appl. Phys. 37, L1362 (1998).

[2] Y. H. Cho. F. Fedler, R. J. Hauenstein, G. H. Park, J. J. Song, S. Keller, U. K. Mishra, and S. P. DenBaars, J. Appl. Phys. 85, 3006 (1999).

[3] T. Wang, H. Saeki, J. Bai, T. Shirahama, M. Lachab, S. Sakai, and P. Eliseev, Appl. Phys. Lett. 76, 1737 (2000).

[4] Y. S. Lin, K. J. Ma, C. Hsu, Y. Y. Chung, C. W. Liu, S. W. Feng, Y. C. Cheng, M. H. Mao, C. C. Yang, H. W. Chuang, C. T. Kuo, J. S. Tsang, and T. E. Weirich, Appl. Phys. Lett. 80, 2571 (2002). 